\title{
An Application of the Finite Element Approximation Method to Find the Complex Zeros of the Modified Bessel Function $K_{n}(z)$
}

\author{
By K. V. Leung and S. S. Ghaderpanah*
}

\begin{abstract}
Using a finite element approximation, an iterative optimization scheme is described to find the $z$ zeros of $K_{n}(z)$ for fixed order $n$. Two computer programs have been implemented to find the complex zeros with a computational accuracy of either 13 or 27 significant digits. The optimization scheme described in the paper may also be readily applied to find real and complex zeros of an arbitrary function with real and complex coefficients. Neither its accuracy nor its efficiency is affected by the number of the roots of the function.
\end{abstract}

1. Introduction. In investigation of wave propagation and scattering in an elastic medium, the modified Bessel functions of the second kind $K_{n}(z)$ are used to describe the potentials for outgoing radiation waves which decay with distance from their source. If scattering problems are studied by use of integral transforms, it becomes necessary to determine the $z$ zeros of $K_{n}(z)$ in order to locate the poles required for inversion of the transform.

Luke [7] evaluated the zeros of Bessel functions by means of rational approximations. Such approximations have proven useful, both as first approximations of the zeros and as the starting point for iterative schemes that use higher-order rational approximations to yield more accurate estimates of the zeros. The error in the rational approximations for $K_{n}(z)$ is treated by Fields [3] and Luke [8] , [9].

Other methods have also been employed successfully [12]. Olver [10] evaluated zeros of Bessel functions of large order using uniform asymptotic expansions. For the case of Hankel functions, this work has been extended by Cochran and Hoffspiegel [1]. Based on the McMahon and Olver expansions, Döring [2] derived a method for the evaluation of complex zeros of cylinder functions.

By tracing the argument of $K_{n}(z)$ along the four sides of a square, and using a double linear interpolation, Parnes [12] has given an iterative scheme to obtain the complex $z$ zeros of $K_{n}(z)$ for fixed integer orders $n=2,3, \ldots, 10$. The scheme consists of generating a sequence of squares whose sides are reduced by a reduction factor of 0.1 at each iteration. If the double linear interpolation could ensure that a complex

Received February 28, 1978; revised August 21, 1978 and December 8, 1978.

AMS (MOS) subject classifications (1970). Primary 30A08, 33A40; Secondary 35L05, $41 \mathrm{~A} 10,65 \mathrm{~N} 30$.

Key words and phrases. Bessel functions, complex zeros, finite element approximation.

*This work is dedicated to the memory of the late Dr. K. V. Leung. 
zero would always be within these squares, each reduction in the size of the square would yield a further significant figure in the location of the zeros.

In the following section a finite element method for finding the complex $z$ zeros of $K_{n}(z)$ is proposed. The method compares favorably with Parnes's iterative scheme insofar as its rate of convergence, computational accuracy and efficiency are concerned. It also compares favorably with other iterative methods such as Ward's downhill method [14] and Frank's quadratic approximation method [4].

2. Formulas and Method. The modified Bessel function of the second kind is defined as follows:

$K_{n}(z)=\frac{1}{2} \sum_{r=0}^{n-1}(-1)^{r} \frac{(n-r-1) !}{r !}\left(\frac{2}{z}\right)^{n-2 r}$

$$
+(-1)^{n+1} \sum_{r=0}^{\infty} \frac{1}{r !(n+r) !}\left(\frac{z}{2}\right)^{n+2 r}\left\{\ln \left(\frac{z}{2}\right)-\frac{1}{2}[\Psi(r+1)+\Psi(n+r+1)]\right\},
$$

where

$$
\Psi(n+r+1)=1+\frac{1}{2}+\frac{1}{3}+\cdots+\frac{1}{n+r}-\gamma \text { and } \Psi(1)=-\gamma
$$

with

$$
\begin{aligned}
\gamma & =\lim _{m \rightarrow \infty}\left[\sum_{k=1}^{m} \frac{1}{k}-\ln (m)\right] \\
& =0.5772156649015328606065120900824024310421 \ldots
\end{aligned}
$$

Let

$$
F\left(x_{1}, x_{2}\right)=\left[R\left(x_{1}, x_{2}\right)\right]^{2}+\left[I\left(x_{1}, x_{2}\right)\right]^{2} \geqslant 0
$$

where $R\left(x_{1}, x_{2}\right)$ and $I\left(x_{1}, x_{2}\right)$ are, respectively, the real and imaginary parts of $K_{n}(z)$ with $z=x_{1}+i x_{2}$.

The problem of finding the $z$ zeros of $K_{n}(z)$ may be reduced to determination of $x_{1}$ and $x_{2}$ to minimize $F\left(x_{1}, x_{2}\right)$. The proposed method of finding the minimum of $F\left(x_{1}, x_{2}\right)$ is described in Appendix I. This method starts with an arbitrarily chosen initial point $\left(x_{1}^{0} x_{2}^{0}\right)$ and generates a sequence of further points $\left(x_{1}^{1}, x_{2}^{1}\right), \ldots$, $\left(x_{1}^{n}, x_{2}^{n}\right), \ldots$, that converges to a point where $F\left(x_{1}, x_{2}\right)$ is minimal. At each iteration $F\left(x_{1}, x_{2}\right)$ is approximated by a quadratic function $F^{*}\left(x_{1}, x_{2}\right)$, and the next point of the sequence is chosen as the point where $F^{*}\left(x_{1}, x_{2}\right)$ is minimal.

3. Results and Observations. All calculations were undertaken on a CDC 6400 computer. A Fortran program, using double-precision arithmetic, was able to perform the calculations to an accuracy of 27 significant figures. However, as complex doubleprecision operations are not available in standard programming languages, the real and 
imaginary parts of $K_{n}(z)$ had to be evaluated by the following formulas:

Real:

$$
\begin{aligned}
R(z)= & \frac{1}{2} \sum_{r=0}^{n-1}(-1)^{r} \frac{(n-r-1) !}{r !}\left(\frac{\rho}{z}\right)^{2 r-n} \cos (2 r-n) \theta \\
& +(-1)^{n+1} \sum_{r=0}^{\infty} \frac{1}{r !(r+n) !}\left(\frac{\rho}{2}\right)^{2 r+n} \\
& \cdot\left\{\left[\ln \left(\frac{\rho}{2}\right)-\frac{\psi(r+1)}{2}-\frac{\psi(r+n+1)}{2}\right] \cos (2 r+n) \theta-\theta \sin (2 r+n) \theta\right\},
\end{aligned}
$$

Imaginary:

$$
\begin{aligned}
I(z)= & \frac{1}{2} \sum_{r=0}^{n-1}(-1)^{r} \frac{(n-r-1) !}{r !}\left(\frac{\rho}{2}\right)^{2 r-n} \sin (2 r-n) \theta \\
& +(-1)^{n+1} \sum_{r=0}^{\infty} \frac{1}{r !(r+n) !}\left(\frac{\rho}{2}\right)^{2 r+n} \\
& \cdot\left\{\left[\ln \left(\frac{\rho}{2}\right)-\frac{\psi(r+1)}{2}-\frac{\psi(r+n+1)}{2}\right] \sin (2 r+n) \theta+\theta \cos (2 r+n) \theta\right\},
\end{aligned}
$$

where $\rho$ and $\theta$ are the modulus and argument of $z$, respectively. The complex zeros of $K_{n}(z)$ were determined for integer order $n=2(1) 10$ and are shown in Table II. At these zeros, $F\left(x_{1}, x_{2}\right)$, as defined by (2), is of the order of $10^{-55}$.

TABLE I

Coordinates of the nodes, and the values of $\alpha_{0}, \alpha_{1}, \alpha_{2}$ at these nodes

\begin{tabular}{|c|c|c|c|c|c|}
\hline$e^{(i, j)}$ & $x_{1}^{(i, j)}$ & $x_{2}^{(i, j)}$ & $\alpha_{0}^{(i, j)}$ & $\alpha_{1}^{(i, j)}$ & $\alpha_{2}^{(i, j)}$ \\
\hline$e^{(0,0)}$ & $x_{1}^{(0,0)}=x_{1}^{x}-\frac{1}{3} h^{r}$ & $x_{2}^{(0,0)}=x_{2}^{x}-\frac{1}{3} h^{x}$ & 1 & 0 & 0 \\
$e^{(1,1)}$ & $x_{1}^{(1,1)}=x_{1}^{(0,0)}+h^{r}$ & $x_{2}^{(1,1)}=x_{2}^{(0,0)}$ & 0 & 1 & 0 \\
$2^{(2,2)}$ & $x_{1}^{(2,2)}=x_{1}^{(0,0)}$ & $x_{2}^{(2,2)}=x_{2}^{(0,0)}+h^{r}$ & 0 & 0 & 1 \\
$e^{(0,1)}$ & $x_{1}^{(0,1)}=x_{1}^{(0,0)}+\frac{1}{2} h^{r}$ & $x_{2}^{(0,1)}=x_{2}^{(0,0)}$ & $\frac{1}{2}$ & $\frac{1}{2}$ & 0 \\
$e^{(0,2)}$ & $x_{1}^{(0,2)}=x_{1}^{(0,0)}$ & $x_{2}^{(0,2)}=x_{2}^{(0,0)}+\frac{1}{2} h^{x}$ & $\frac{1}{2}$ & 0 & $\frac{1}{2}$ \\
$e^{(1,2)}$ & $x_{1}^{(1,2)}=x_{1}^{(0,0)}+\frac{1}{2} h^{r}$ & $x_{2}^{(1,2)}=x_{2}^{(0,0)}+\frac{1}{2} h^{r}$ & 0 & $\frac{1}{2}$ & $\frac{1}{2}$ \\
\hline
\end{tabular}

It appears that the required number of iterations, and hence the amount of CPU time required to obtain the zeros of $K_{n}(z)$, is very sensitive to changes in the reduction factor $\lambda$ of the size of the finite elements. Starting from an initially chosen point where $x_{1}=-1.3$ and $x_{2}=0.5$, and with values of the reduction factor chosen as 


\section{TABLE II}

The real parts $R(z)$ and imaginary parts $I(z)$ of the complex zeros of $K_{n}(z)$ with $n=2(1) 10$

$\begin{array}{rcc}n & R(z) & \pm I(z) \\ 2 & -1.28137379765609647788484347943 & 4.2948496520871968779577284110 \\ 3 & -1.6817888047458454647221805246 & 1.3080120322739490396502284389 \\ 4 & -1.9781618634659070241700412324 & 2.2043719815468711802301895462 \\ 4 & -2.6286711679571242189239356978 & 4.3269664862177846518524769588 \\ 5 & -2.2186262746398760466991398972 & 3.1130829449859484932024599078 \\ 5 & -3.1351328447046434221402224695 & 1.3038823977137057333868902412 \\ 6 & -2.4234043880011252372669320326 & 4.0309615812693082518160866112 \\ 6 & -3.5510979040000786787689256194 & 2.1834951775778858208942180135 \\ 6 & -3.9615580702543404860650878072 & 4.3334540861473783228197493649 \\ 7 & -2.6031262658681676073605916898 & 4.9559696065385237377946547292 \\ 7 & -3.9081257398031834786414725236 & 3.0708717702488955667126782799 \\ 7 & -4.5126267774997091289243634516 & 1.3027788416202445155997188405 \\ 8 & -2.7641429773113422214747067049 & 5.8867128822557099755500071045 \\ 8 & -4.2231522789550338555580428982 & 3.9650659693874916655430042023 \\ 8 & -4.9882787925531101790986431543 & 2.1770827464789907589102679543 \\ 8 & -5.2907612925994479853857895222 & 4.3357769522400748475850727130 \\ 9 & -2.9105824231361973641127863752 & 6.8221903314329512927258712281 \\ 9 & -4.5064659455202975444947754224 & 4.8652071432637217649541918277 \\ 9 & -5.4097474475400236316635820253 & 3.0565442393434597827549120928 \\ 9 & -5.8665514666851772591442131577 & 1.3023283269981807534615329324 \\ 10 & -3.0452934989589489096726573840 & 7.7616556708745682219592817911 \\ 10 & -4.7648453733729039927675961572 & 5.7705555987099768475527429057 \\ 10 & -5.7900271641796772769369101588 & 3.9409726157692468366207406587 \\ 10 & -6.3783949707941569023306522990 & 2.1742485862026654802191073802 \\ 10 & -6.6184818847079360692456212008 & 4.3368620578620986460836833209\end{array}$

$0.5,0.25,0.025$, and 0.0001 , a zero of $K_{n}(z)$ is found after $45,23,13$, and 7 iterations, respectively. The effects of the reduction factor $\lambda$ on the number of iterations and CPU time are shown in Figures II and III.

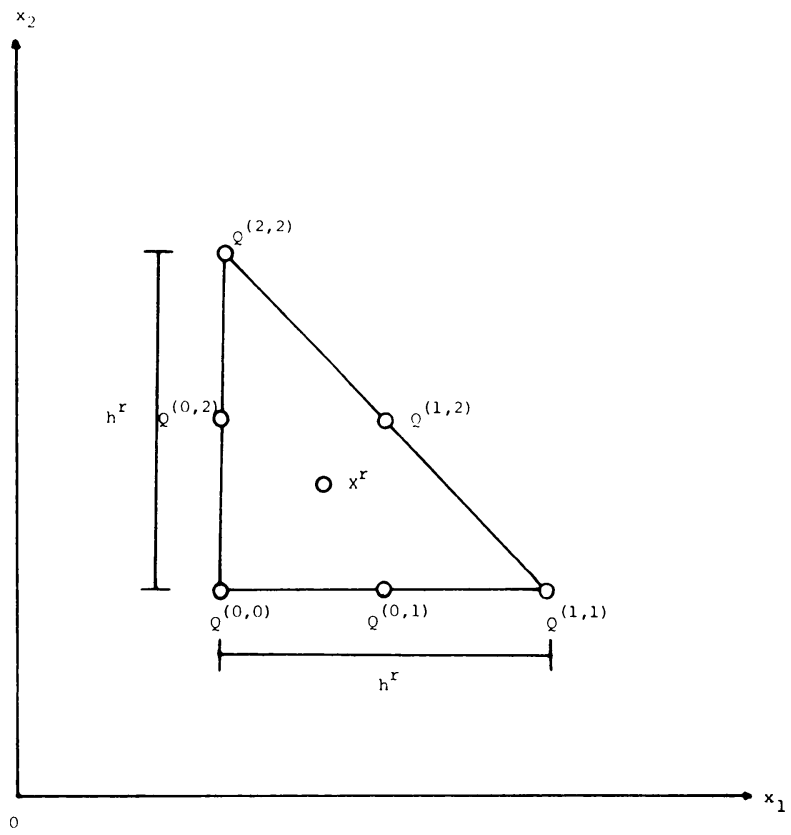

FIGURE 1

Finite right-angled triangular element $\Delta^{r}$ associated with $X^{r}=\left(x_{1}^{r}, x_{2}^{r}\right)$ 


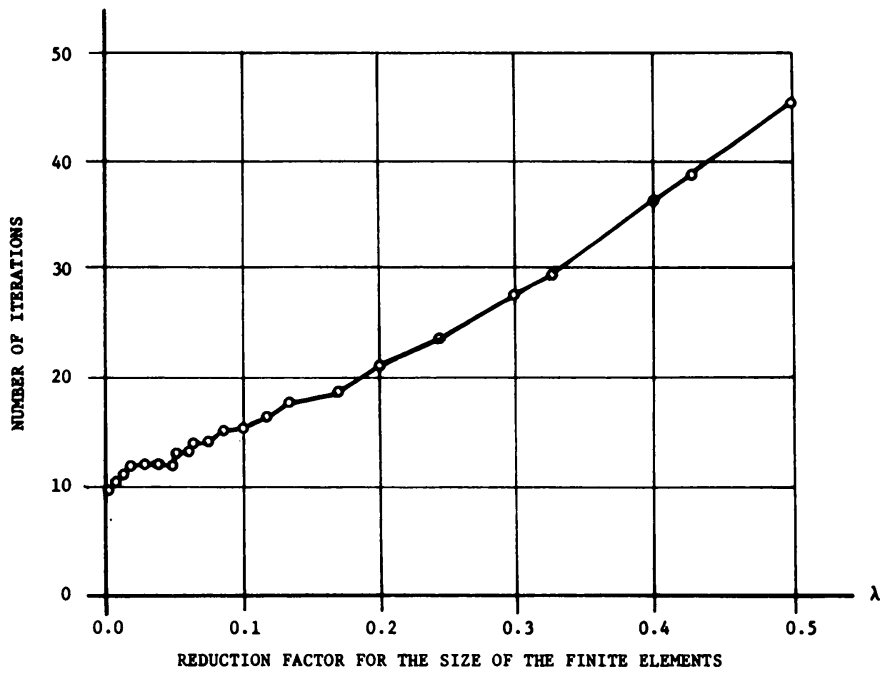

Figure II

Dependence of number of iterations on the reduction factor for the step size of the finite element

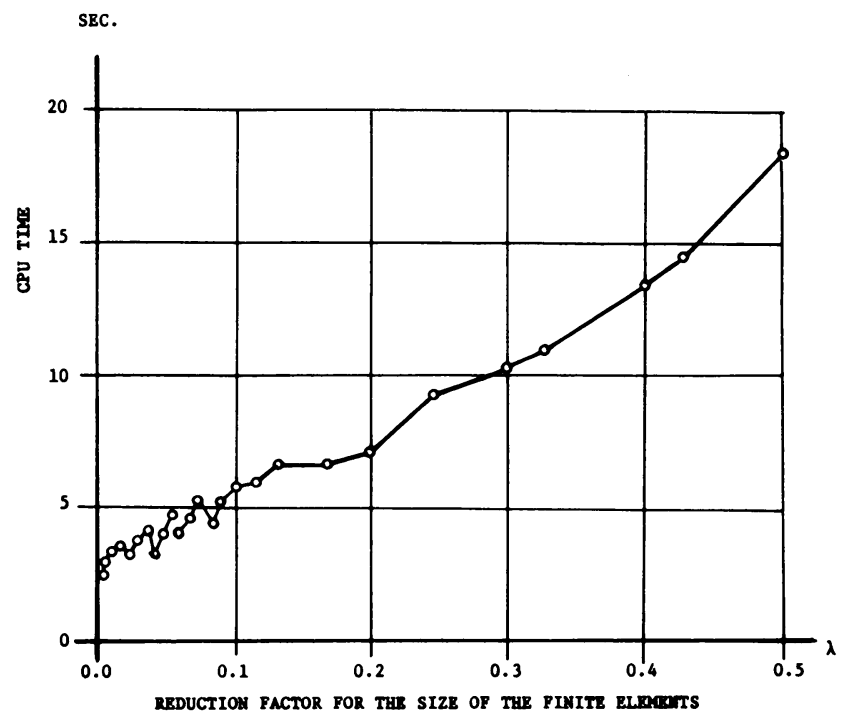

FIGURE III

Dependence of CPU time on the reduction factor for the step size of the finite element

A second computer program, also written in Fortran, but using single-precision arithmetic has been tested. This program, which is four times faster than the previous version, provides computations to only 13 significant digits.

Acknowledgments. This research was supported by National Research Council of Canada under grant No. A4342. The author wishes to sincerely thank Professor H. S. Heaps for his helpful comments and suggestions for improvements. I would also like to thank M. Pierotti for the typing. 


\section{Appendix I}

The Finite Element Algorithm. The algorithm described here is based on a sequence of quadratic surface-fitting problems. At each stage of minimization, the objective function $F\left(x_{1}, x_{2}\right)$ is approximated by a quadratic form over the finite element region about the current minimum point. Zlamal [16] has proved the existence and uniqueness of such a quadratic polynomial. Each finite element is a rightangle triangle, with nodes at the vertices and midpoints of each side. Ghaderpanah [5] has generalized this technique and applied it in higher-dimensional minimization problems.

Iteration Steps. 1. Set $r=0$. A starting point $X^{0}=\left(x_{1}^{0}, x_{2}^{0}\right)$ and a step size $h^{0}$ are given.

2. Select the vertices $Q^{(i, i)}, i=0,1,2$, for the right-angle triangle $\Delta^{r}$ according to the following scheme:

$$
\begin{aligned}
& Q^{(0,0)}=X^{r}-\left(\frac{h^{r}}{3}, \frac{h^{r}}{3}\right) \\
& Q^{(1,1)}=\left(q_{1}^{(0,0)}+h^{r}, q_{2}^{(0,0)}\right) \\
& Q^{(2,2)}=\left(q_{1}^{(0,0)}, q_{2}^{(0,0)}+h^{r}\right)
\end{aligned}
$$

and determine the side midpoints as follows:

$$
Q^{(1, k)}=1 / 2\left[Q^{(1,1)}+Q^{(k, k)}\right], \quad 0 \leqslant 1<k<2 .
$$

Figure I illustrates the construction of the element about the point $\boldsymbol{X}^{\boldsymbol{r}}$.

3. Compute the nodal values $F^{(i, j)}$ at the six nodes $Q^{(i, j)}$, i.e.,

$$
F^{(i, j)}=F\left(Q^{(i, j)}\right), \quad 0 \leqslant i \leqslant j \leqslant 2 .
$$

4. Approximate $F$ by the quadratic $F^{*}$ given by

$$
F^{*}(X)=\sum_{i=0}^{2} \sum_{j=i}^{2} F^{(i, j)} S^{(i, j)}\left(\alpha_{i}, \alpha_{j}\right),
$$

where $S^{(i, j)}, 0<i \leqslant j \leqslant 2$, are the quadratic shape functions defined by

$$
S^{(i, j)}\left(\alpha_{i}, \alpha_{j}\right)=2\left(2-\delta_{i j}\right) \alpha_{i} \alpha_{j}-1 / 2 \delta_{i j}\left(\alpha_{i}+\alpha_{j}\right)
$$

and $\delta_{i j}$ is the Kronecker delta. The functions $\alpha_{i}$ are the coordinate functions defined by

$$
\alpha_{i}(X)=\frac{1}{h^{r}}\left(x_{i}-q_{i}^{(0,0)}\right), \quad i=1,2, \quad \text { and } \quad \alpha_{0}=1-\alpha_{1}-\alpha_{2} .
$$

It is easy to verify that the functions $S^{(i, j)}$ and $\alpha_{i}$ satisfy the respective properties of shape functions and natural coordinate functions given in [15]. The values of $\alpha_{i}$ at each node are explicitly shown in Table I. It may be shown that the quadratic $F^{*}$ is continuous and interpolates $F$ at the six nodes $Q^{i, j}$, i.e.,

$$
F^{*}\left(Q^{(i, j)}\right)=F\left(Q^{(i, j)}\right), \quad 0 \leqslant i \leqslant j \leqslant 2 .
$$


Moreover, the first and second partial derivatives of $F$ and $F^{*}$ at $Q^{(0,0)}$ are identical. Therefore, the approximation is of second order over the triangular region, thus

$$
\left|F(X)-F^{*}(X)\right| \approx O\left(\left(h^{r}\right)^{3}\right)
$$

for all $X$ in $\Delta^{r}$.

5. Determine the new approximation to the local minimum $X^{r+1}$ as follows:

$$
x_{i}^{r+1}=q_{i}^{(0,0)}+\alpha_{i}^{r} h^{r}, \quad i=1,2,
$$

where $\left(\alpha_{0}^{r}, \alpha_{1}^{r}, \alpha_{2}^{r}\right)$ is a stationary of $F^{*}$ in the natural coordinate system obtained by solving the system of algebraic equations in the unknowns $\alpha_{0}^{r}, \alpha_{1}^{r}$ and $\alpha_{2}^{r}$ given by

$$
\frac{\partial F^{*}}{\partial \alpha_{1}^{r}}=0, \quad \frac{\partial F^{*}}{\partial \alpha_{2}^{r}}=0, \quad \alpha_{0}^{r}+\alpha_{1}^{r}+\alpha_{2}^{r}=1 .
$$

The solution of this system is given by:

$$
\alpha_{1}^{r}=\left(B_{1} A_{22}-B_{2} A_{12}\right) / D, \quad \alpha_{2}^{r}=\left(B_{2} A_{11}-B_{1} A_{21}\right) / D
$$

and $\alpha_{0}^{r}=1-\alpha_{1}-\alpha_{2}$, where $D=A_{11} A_{22}-A_{12} A_{21}$ and

$$
\left\{\begin{array}{l}
A_{11}=F^{(0,0)}-2 F^{(0,1)}+F^{(1,1)} \\
A_{22}=F^{(0,0)}-2 F^{(0,2)}+F^{(2,2)} \\
A_{12}=A_{21}=F^{(0,0)}-F^{(0,1)}-F^{(0,2)}+F^{(1,2)}, \\
B_{1}=3 / 4 F^{(0,0)}-F^{(0,1)}+1 / 4 F^{(1,1)} \\
B_{2}=3 / 4 F^{(0,0)}-F^{(0,2)}+1 / 4 F^{(2,2)}
\end{array}\right.
$$

Let $\delta^{r}$ be the distance between the solution $\bar{X}=\left(\bar{x}_{1}, \bar{x}_{2}\right)$ and the point $X^{r}=\left(x_{1}^{r}, x_{2}^{r}\right)$, i.e., $\delta^{r}=\left\|X^{r}-\bar{X}\right\|$. It may be shown by use of the Taylor series expansion of $F$ that for sufficiently small $\delta^{r}, \delta^{r+1}$ is reduced to $O\left(\left(\delta^{r}\right)^{2}\right)$.

6. Termination Criterion and Convergence. Let $0<\lambda<1$, be a predetermined reduction factor for the step size. Define the step size $h^{r+1}$ for the next iteration as follows:

$$
h^{r+1}= \begin{cases}\lambda h^{r} & \text { if } X^{r} \text { is inside } \Delta^{r}, \text { i.e., } 0 \leqslant \alpha_{0}^{r}, \alpha_{1}, \alpha_{2}^{r} \leqslant 1, \\ h^{r} & \text { if otherwise. }\end{cases}
$$

Terminate the process if $h^{r+1}<\epsilon_{1}$ or

$$
F\left(X^{r+1}\right)-F\left(X^{r}\right)<\epsilon_{2} \text { and }\left\|X^{r+1}-X^{r}\right\|<\epsilon_{3},
$$

where the nonzero scalars $\epsilon_{1}, \epsilon_{2}$ and $\epsilon_{3}$ are predetermined minimal improvement, minimal step length and minimal step size, respectively. Otherwise, accept the new point $X^{r+1}$, set $r=r+1$ and repeat from step 2 . 
1. J. A. COCHRAN \& J. N. HOFFSPIEGEL, "Numerical techniques for finding $v$-zeros of Hankel functions," Math. Comp., v. 24, 1970, pp. 413-422. MR 42 \#7038.

2. B. DÖRING, "Complex zeros of cylinder functions," Math. Comp., v. 20, 1966, pp. 215 -222. MR 33 \#857.

3. J. L. FIELDS, "A linear scheme for rational approximations," J. Approximation Theory, v. 6, 1972, pp. 161-175.

4. W. L. FRANK, "Finding zeros of arbitrary functions," J. Assoc. Comput. Mach., v. 5, 1958 , pp. $154-160$.

5. S. S. GHADERPANAH, Some Applications of Finite Element Method, Chapter I, Master Thesis, Dept. of Comp. Sci., Concordia University, 1979.

6. K. V. LEUNG, H. S. HEAPS \& D. JEFFRIES, "Model fitting process for enzyme systems with time-lag,"Proc. Third Internat. Cong. Cybernetics and Systems, Bucharest, 1975.

7. Y. L. LUKE, "The special functions and their approximation," Vol. II, Math. in Sci. and Engineering, v. 53, Academic Press, New York, 1969. MR 39 \#3039.

8. Y. L. LUKE, Mathematical Functions and their Approximations, Academic Press, New York, 1975.

9. Y. L. LUKE, Algorithms for the Computation of Mathematical Functions, Academic Press, New York, 1977.

10. F. W. J. OLVER, "The asymptotic expansion of Bessel functions of large order," Philos. Trans. Roy. Soc. London Ser. A, v. 247, 1954, pp. 328-368. MR 16, 696.

11. M. N. OGUZTÖRELI, K. V. LEUNG \& D. L. JEFFRIES, “A kinetic study of enzyme systems," Nonlinear Analysis: Theory, Methods and Applications, v. 1, Pergamon Press, New York, 1976, pp. 15-35.

12. R. PARNES, "Complex zeros of the modified Bessel function $K_{n}(Z)$," Math. Comp., v. 26, 1972, pp. 949-953.

13. D. W. SWEENEY, "On the computation of Euler's constant," Math. Comp., v. 17, 1963, pp. 170-178. MR 28 \#3522.

14. J. A. WARD, "The down-hill method of solving $f(z)=0, " J$. Assoc. Comput. Mach., v. 4,1957, pp. $148-150$.

15. O. C. ZIENKIEWICZ, The Finite Element Method in Engineering Science, McGrawHill, New York, 1971.

16. M. ZLÁMAL, "On the finite element method," Numer. Math., v. 12, 1968, pp. 394409. MR 39 \#5074. 